\title{
Metallic Glass / PVDF Magnetoelectric Laminates for Resonant Sensors and Actuators
}

\author{
Jon Gutiérrez ${ }^{1, *}$, Andoni Lasheras ${ }^{2}$, Pedro Martins ${ }^{3}$, Nélson Pereira ${ }^{3}$, Jose M. Barandiarán ${ }^{1}$ \\ and Senentxu Lanceros-Mendez ${ }^{1,4}$
}

1 BCMaterials and Universidad del País Vasco UPV/EHU, P.O. Box 644, 48080 Bilbao, Spain; E-Mails: jon.gutierrez@ehu.eus (J.G.); manu@bcmaterials.net (J.M.B.); senentxu.lanceros@bcmaterials.net (S. L.-M.);

2 Departamento de Electricidad y Electrónica, Universidad del País Vasco UPV/EHU, P.O. Box 644, 48080 Bilbao, Spain; E-Mails: andoni.lasheras@ehu.es (A.L.);

3 Centro/Departamento de Física, Universidade do Minho, 4710-057 Braga, Portugal; E-Mails: pmartins@fisica.uminho.pt (P. M.);nmmsp.18@gmail.com (N.P.);

4 IKERBASQUE, Basque Foundation for Science, 48013 Bilbao, Spain;

E-Mails: senentxu.lanceros@bcmaterials.net (S. L.-M.);

* Author to whom correspondence should be addressed; E-Mail: jon.gutierrez@ehu.eus (J.G.); Tel.: +34-94-601-2553; Fax: +34-94-601-3500

\begin{abstract}
Among magnetoelectric (ME) heterostructures, $\mathrm{ME}$ laminates of the type Metglas-like / PVDF (magnetostrictive+piezoelectric constituents) have shown the highest induced $\mathrm{ME}$ voltages, usually detected at the magnetoelastic resonance of the magnetostrictive constituent. This ME coupling happens because of the high crosscorrelation coupling between magnetostrictive and piezoelectric material, and is usually associated with a promising application scenario for sensors or actuators. In this work we detail the basis of the operation of such devices, as well as some arising questions (as size effects) concerning their best performance. Also, some examples of their use as very sensitive magnetic fields sensors or innovative energy harvesting devices will be presented At the end, the challenges, future perspectives and technical difficulties that will determine the success of ME composites for sensor applications are discussed.
\end{abstract}

Keywords: magnetoelectrics; magnetoelectric heterostructures; magnetoelectric sensors;

\section{Introduction}

The magnetoelectric (ME) effect is defined as the electrical field (or voltage) induced under the application of a magnetic field (direct ME effect), or vice versa, as the magnetic induction induced under the application of an electrical field (inverse ME effect). In 1894 the intrinsic ME effect was 
theoretically predicted by Pierre Curie, and was experimentally first observed nearly 60 years ago in single-[1] and poly-crystals [2] of single-phased materials, but it turn out to be a weak effect observed only at low temperatures. To make the magnitude of this effect useful for applications, a good alternative was to convert the known magnetoelectric materials to composite systems in which one of the constituent was purely magnetic/magnetostrictive and the other one, purely piezoelectric. These composite heterostructures were of the type "particulate" and "laminate" ones. The first particulate composites were made of magnetostrictive ferrites and piezoelectric $\mathrm{Pb}(\mathrm{Zr}, \mathrm{Ti}$ ) $\mathrm{O} 3$ (or $\mathrm{PZT}$ ) and gave values of the induced magnetoelectric voltages up to $0.4 \mathrm{~V} / \mathrm{cm}$.Oe [3,4]. However, these new hybrid systems exhibited some problems as chemical reactions between the starting materials during the sintering process, or mechanical defects that limited the mechanical coupling between the particles of the constituents [5].

These problems were overcome in 2001 by using laminate composites, with layers of magnetostrictive and piezoelectric phases epoxied together. Ryu et al [6] reported a magnetoelectric voltage of $4.68 \mathrm{~V} / \mathrm{cm}$. Oe in an structure consisting in a disc of PZT sandwiched between two discs of Terfenol-D. Nevertheless, despite the good performance of this type of magnetoelectric laminates they were still some problems remaining, as brittleness, low permeability and the high applied magnetic fields needed to achieve the maximum ME effect for the magnetostrictive phase (mostly Terfenol-D), and also brittleness and high resistance of the piezoelectric component (mostly PZT) to reduce eddy current losses of the composite.

New combinations of magnetostrictive/piezoelectric layers were needed; thus, by using high permeability magnetostrictive materials such as iron-based Metglas alloys epoxied to poly(vinylidene fluoride)(PVDF) piezoelectric polymer [7], signals as high as $7.2 \mathrm{~V} / \mathrm{cm}$.Oe at low (sub-resonant) frequency and $310 \mathrm{~V} / \mathrm{cm}$.Oe at the electromechanical resonance of the composite, were obtained. This electromechanical resonance takes place when a mechanical resonant response is excited through the magnetostrictive effect of the magnetic constituent of the laminate, or what is equivalent at its corresponding magnetoelastic resonance (MER) frequency.

To account for such results, we have to analyze the ME effect magnitude, that is usually defined as the product between the piezomagnetic and piezoelectric effects [8]:

$$
\alpha_{M E}=\frac{d E}{d H}=k_{c}\left(\frac{\delta \lambda}{\delta H}\right)\left(\frac{\delta E}{\delta \lambda}\right)
$$

for laminates with unconstrained longitudinal vibration. In this equation $\delta \lambda / \delta H$ is the piezomagnetic coefficient of the magnetic element, $\delta E / \delta \lambda$ is the piezoelectric constant of the dielectric one and $k_{c}$ is the coupling constant (arisen from bonding conditions) between both constituents. So, even if magnetostriction of Terfenol-D, $\lambda>1000 \mathrm{ppm}$, is much higher than the magnetostriction of a Metglaslike amorphous magnetic material with $\lambda \approx 40 \mathrm{ppm}$ in the best cases, the quantity that drives the ME is actually the piezomagnetic coefficient of the magnetic constituent, $\delta \lambda / \delta H=d_{33}^{m}$; while for Terfenol-D the maximum value of $d_{33}^{m}=1,2 \times 10^{-6} / \mathrm{Oe}$ occurs at about 500 Oe applied external magnetic field [9], for commercial or home-made Metglas-like amorphous materials this maximum $d_{33}^{m}$ value is almost equal, but it can be achieved at an applied field of only a few Oe.

Among the piezoelectric compounds some specific functional polymers such as Poly(vinylidine Fluoride) (PVDF) polymer and its copolymer Poly(vinylidene fluride/trifluoroethylene) P(VDF-TrFE) have been widely used and optimized as piezoelectric matrix on ME structures due to their interesting 
ferroelectric and piezoelectric properties [10]. They have a moderate piezoelectric coefficient of a few $\mathrm{pC} / \mathrm{N}$, but they show the advantages of being strongly flexible as well as used in film form, which makes it very useful to conform surfaces of different shape. Notwithstanding the higher ME coefficients being reported on piezoelectric ceramic-based composites, polymer-based ME composites offer more simple elaboration process, absence of brittleness and fragility, low electrical resistivity and high dielectric losses.

The mechanism underlying the ME effect in laminates is easy to understand: the magnetostrictive constituent will deform under the action of an applied external magnetic field, H. This strain will transmit to the piezoelectric material layer through elastic bonding with an epoxy between both constituents. Finally, this deformation of the piezoelectric material will give rise to an induced ME voltage through piezoelectricity.

The close collaboration among the BCMaterials Research Center, the Group of Magnetism and Magnetic Materials of the Universidad del País Vasco (UPV/EHU), both from the Basque Country, Spain, and the Electroactive Smart Materials Group from the Universidade do Minho at Braga and Guimaraes, Portugal, has given as result an intense and fruitful activity devoted to the development of Metglas-like/PVDF laminated magnetoelectric composites with high response that have been tested for high sensitivity sensors and actuators based on the previously mentioned effect. In the following, we present the relevant background to the polymer-based ME laminates fabrication and characterization methods and discuss the key considerations in the selection of materials and in the design of these ME devices. We summarize our results concerning magnetic sensors and energy harvesters based on Metglas-like/PVDF ME laminates, as well as address the mains challenges and prospective for the near future.

\section{Experimental}

\subsection{Materials: magnetostrictive and piezoelectric constituents}

In which concerns the magnetostrictive constituent, all metallic glasses that appear in this work belong to one of these: either commercial Vitrovac $4040\left(\mathrm{Fe}_{39} \mathrm{Ni}_{39} \mathrm{Mo}_{4} \mathrm{Si}_{6} \mathrm{~B}_{12}\right)$ or home-made samples with nominal compositions $\left(\mathrm{Fe}_{0.79} \mathrm{Co}_{0.21}\right)_{75+\mathrm{x}} \mathrm{Si}_{15-1.4 \mathrm{x}} \mathrm{B}_{10+0.4 \mathrm{x}}(\mathrm{X}=0,3,6)$ and $\mathrm{Fe}_{85-\mathrm{x}} \mathrm{Co}_{\mathrm{x}} \mathrm{B}_{15}$ with $\mathrm{X}=21$. All these are Fe-based metallic glasses containing Fe-Co-Ni-Si-B in their composition and were prepared by the single roller quenching method in the form of long ribbons. Different pieces of the same ribbon were cut to perform their magnetic and magnetoelastic characterization. Room temperature hysteresis loops were measured by a classical induction method, obtaining so saturation magnetization $\left(\mu_{o} M_{s}\right)$ and susceptibility $(\chi)$ values. Magnetostriction $(\lambda)$ was determined by using strain gages and from this measurement the piezomagnetic coefficient $d_{33}^{m}=\delta \lambda / \delta H$ was determined. Extensive magnetoelastic resonance measurements have been performed to determine resonant $\left(f_{r}\right)$ and anti-resonant $\left(f_{a}\right)$ frequencies (figure 1a, upper) and the signal amplitude at the resonance for all the studied samples, as it will be extensively explained in the following subsection. Table 1 summarizes the obtained mean magnetic parameters for all the used magnetostrictive alloy compositions [11].

Table 1. Selected magnetic properties of the magnetostrictive sample compositions used in the fabrication of all ME laminates appearing in this work. 


\begin{tabular}{|c|c|c|c|c|}
\hline & $\mu_{0} M_{s}(T)$ & $\chi$ & $\lambda(\mathbf{p p m})$ & $\begin{array}{c}d_{33}^{m}=\delta \lambda / \delta H \\
(\mathbf{p p m} / \mathbf{O e})\end{array}$ \\
\hline & \multicolumn{4}{|c|}{$\left(\mathrm{Fe}_{0.79} \mathrm{Co}_{0.21}\right)_{75+\mathrm{x}} \mathrm{Si}_{15-1.4 \mathrm{x}} \mathrm{B}_{10+0.4 \mathrm{x}}$} \\
\hline$X=0$ & 1.3 & 55000 & 18 & 1.4 \\
\hline$X=3$ & 1.4 & 36000 & 20 & 1.5 \\
\hline \multirow[t]{2}{*}{$X=6$} & 1.7 & 50000 & 23 & 2 \\
\hline & \multicolumn{4}{|c|}{$\mathrm{Fe}_{85-\mathrm{x}} \mathrm{Co}_{\mathrm{x}} \mathrm{B}_{15}$} \\
\hline \multirow[t]{3}{*}{$X=21$} & 1.9 & 70000 & 25 & 2.8 \\
\hline & \multicolumn{4}{|c|}{ Vitrovac $4040\left(\mathrm{Fe}_{39} \mathrm{Ni}_{39} \mathrm{Mo}_{4} \mathrm{Si}_{6} \mathrm{~B}_{12}\right)^{*}$} \\
\hline & 0.8 & $>1000$ & 8 & 1.4 \\
\hline
\end{tabular}

*values taken from Ref. [12]

Concerning the piezoelectric polymer we have used PVDF, the well-known piezoelectric polymer $[10,13]$. It shows moderate piezoelectric coefficients, ranging as $d_{33}^{p} \approx 24-34 \mathrm{pC} / \mathrm{N}$ and $d_{31}^{p} \approx 8-22$ $\mathrm{pC} / \mathrm{N}$. In addition, it has glass transition and melting temperatures about $-35{ }^{\circ} \mathrm{C}$ and $171{ }^{\circ} \mathrm{C}$, respectively, but a Curie temperature of $\approx 100{ }^{\circ} \mathrm{C}$.

Figure 1. Geometry of a (a) bilayer MP and (b) three-layer sandwich-like (L-T type) MPM structures. $\mathrm{u}(\mathrm{t})$ is the alternating induced ME voltage. Figure taken with permission from [16].
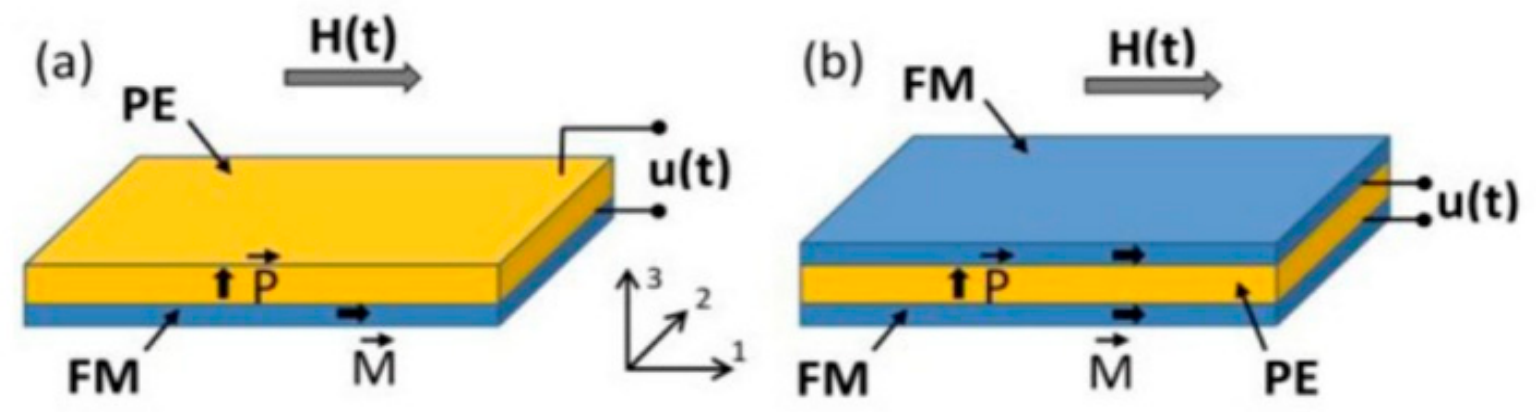

Polymer-based ME laminates are typically produced by epoxing magnetostrictive layers (Metglaslike or Vitrovac; M) to commercial poled $\beta$-PVDF (P), following the optimized conditions presented in previous studies $[14,15]$. All the laminates appearing within this work are bilayers (MP) or three-layer sandwich-like (L-T type, MPM) laminated composites.

\subsection{From the magnetoelastic resonance to the induced magnetoelectric effect}

The Fe-based metallic glasses used in our fabricated ME laminates show an excellent coupling between magnetic and elastic properties, and as well as an applied external magnetic field causes magnetostrictive deformation of the magnetic material, the inverse effect also happens. That is, any application of a mechanical stress causing a deformation in the ferromagnetic material, will cause a change in its magnetic state. This is the so called magnetoelastic or Villari effect. A direct consequence of such magnetoelastic coupling is the dependence of elastic constants of magnetostrictive materials 
with applied external magnetic field, which in the case of magnetostrictive amorphous long ribbons translates to a clear dependence of longitudinal Young's modulus with $H$ or $\Delta E$ effect.

It turns out easy to measure since magnetization changes can be detected inductively; thus, if longitudinal deformations of the metallic glass ribbon piece are excited through magnetostriction, the elastic sound wave induced in the sample will be accompanied by a magnetization one, giving rise to a magnetoelastic wave (a detailed mathematical formalism can be found in [17]).

Driving the induced elastic wave adequately by changing the frequency of the applied external magnetic field, the detected magnetoelastic wave will become stationary and will enter to a resonant state at a resonant frequency $f_{r}$. We have used a home-mounted magnetoelastic resonance detection apparatus that automatically changes the external applied magnetic field (or bias) $H_{d c}$ and the value of the frequency of the $h_{a c}$ magnetic excitation in order to drive the sample to its magnetoelastic resonance at a given $H_{d c}$, and stores the correspondent frequencies for the maximum (or resonant, $f_{r}$ ) and minimum (or anti-resonant, $f_{a}$ ) induced signals, together with the signal amplitude at the resonance $[18,19]$. These measured frequencies and mainly the resonant $\left(f_{r}\right)$ one will vary with the bias field $H$, and so it will do the Young's modulus determined as

$$
E(H)=\left[2 L f_{r}^{2}(H)\right]^{2} \rho
$$

where $L$ and $\rho$ are the length and density of the sample. This field-dependence of this elastic modulus is known as $\Delta E$ effect $\left(\Delta E=1-E(H) / E_{S}, E_{S}\right.$ being the Young's modulus measured at magnetic saturation). Other useful magnetoelastic parameters that can be determined from these measurements are the magnetomechanical coupling coefficient $\left(k=\left(\pi^{2} / 8\right)\left(1-\left(f_{r} / f_{a}\right)^{2}\right)\right)$ and quality factor of the resonance $\left(Q=\Delta f l f_{r}\right)$, all quantities being function of the applied external magnetic field.

Figure 2(a) shows an example of such magnetoelastic resonance measurements performed on a 30 $\mathrm{mm}$ (length) $\times 1.8 \mathrm{~mm}$ (width) $\times 30 \mathrm{~mm}$ (thickness) long ribbon of as-cast $\mathrm{Fe}_{64} \mathrm{Co}_{17} \mathrm{Si}_{6.6} \mathrm{~B}_{12.4}$ amorphous magnetostrictive material. The maximum value of the $\Delta E$ effect occurs at low fields where magnetostriction has not achieved its maximum value (see Figure 2(b)), but yes its derivative the piezomagnetic coefficient $\delta N \delta H$ (inset in Figure 2(b)). Taking into account equation (1), it immediately arises the fact that maximum magnetoelectric induced voltage will be found at the maximum value of $\delta \lambda \delta H$, usually at applied $H_{d c}$ fields close to the corresponding maximum value of the $\Delta E$ effect ( $E(H)$ minimum value) and always at the electromechanical resonance of the laminate composites.

Figure 2. (a) Magnetoelastic resonance and (b) magnetostriction (and its field derivative) measurements performed on a long ribbon of as-cast $\mathrm{Fe}_{64} \mathrm{Co}_{17} \mathrm{Si}_{6.6} \mathrm{~B}_{12.4}$ amorphous magnetostrictive material; (c) ME measurements performed in a three-layer L-T sandwich configuration: the same previous magnetostrictive ribbon is longitudinally magnetized while the piezoelectric polymer (PVDF) is transversely poled. 


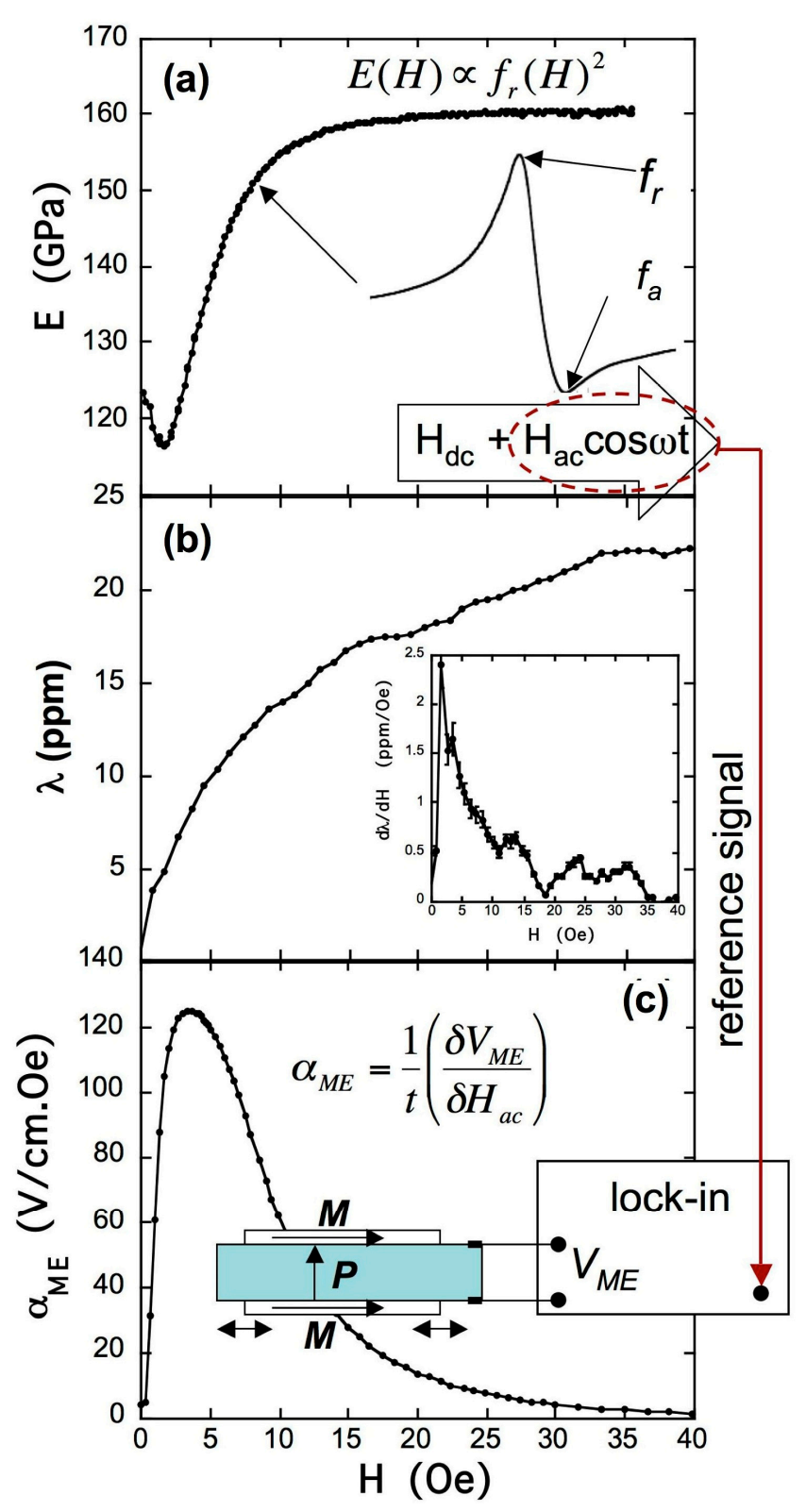

So, in order to measure the ME effect magnitude we only needed to slightly modify our former magnetoelastic resonance apparatus: coaxial solenoids with the ME laminate in its centre apply a net magnetic field $H(t)=H_{d c}+H_{a c} \cos \omega t\left(H_{a c}<<H_{d c}\right)$ on it. We first determine the static field $H_{d c}$ needed for maximum amplitude of the magnetoelastic resonance. Under a $H_{a c}$ magnetic excitation applied along the length axis, the magnetostrictive ribbons will elongate and shrink along that direction. This will make the piezoelectric film of PVDF to undergo an $a c$ longitudinal strain, inducing a dielectric polarization change in its transverse direction that is accurately measured as a magnetoelectric voltage $V_{M E}$ by using a lock-in amplifier (Figure 2(c)). From this voltage the magnetoelectric coefficient $\alpha_{M E}$ can be directly obtained as [6]:

$$
\alpha_{M E}=\frac{d E}{d H}=\frac{1}{t}\left(\frac{\delta V_{M E}}{\delta H_{a c}}\right)
$$

A clear advantage of this measurement method is that the same experimental set-up allows us to determine simultaneously (a) the ME response dependence as the bias field $H_{d c}$ changes and, (b) the magnetoelectric voltage dependence against the applied $H_{a c}$ magnetic excitation, the so called 
sensitivity of the ME laminate.

\section{Some applications of magnetoelectric laminates}

\subsection{Magnetic Field Sensor}

Meeting the nowadays increasing demands for vector magnetometers the anisotropic ME voltage response on a $\mathrm{Fe}_{61.6} \mathrm{Co}_{16.4} \mathrm{Si}_{10.8} \mathrm{~B}_{11.2} / \mathrm{PVDF} / \mathrm{Fe}_{61.6} \mathrm{Co}_{16.4} \mathrm{Si}_{10.8} \mathrm{~B}_{11.2}$ laminate has been utilized for the development of a magnetic field sensor capable to sense the magnitude and direction of both AC and DC magnetic fields (see Figure 3).

Figure 3. a) $\mathrm{ME}$ voltage value variation with the $0-360^{\circ}$ angle range at: a) resonance frequency and b) non-resonance frequency. Figure taken with permission from [20].
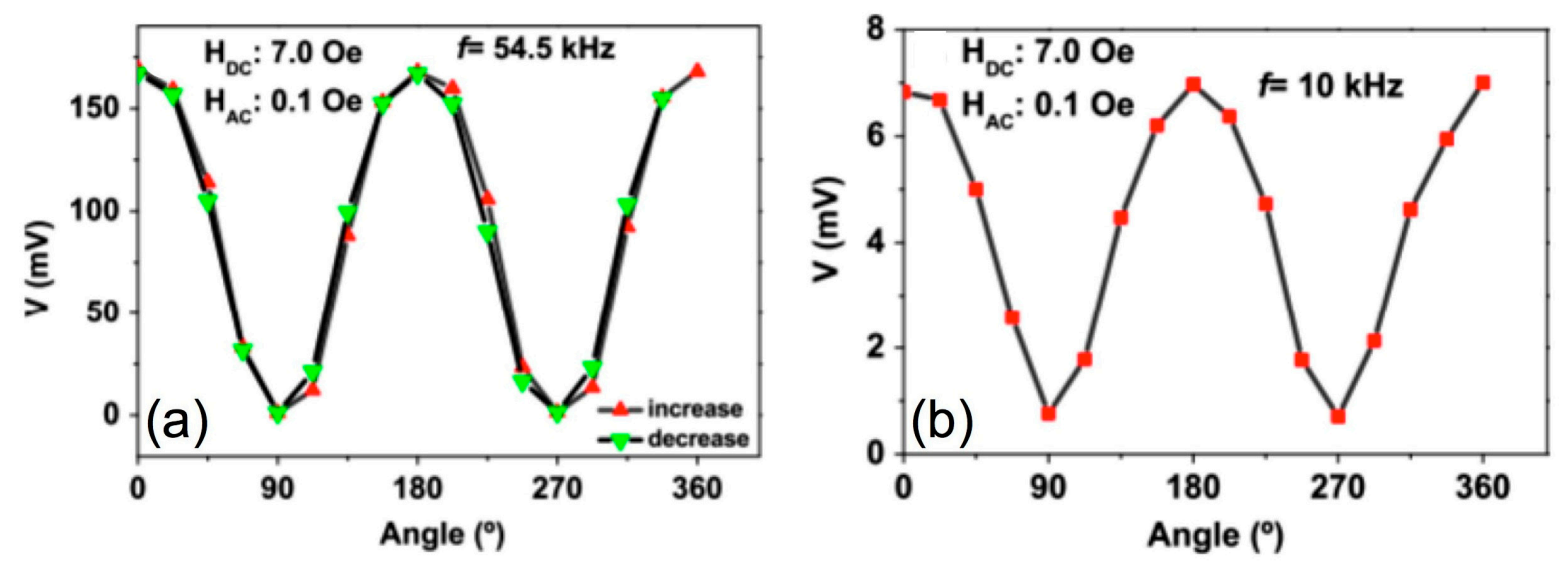

The linearity (92\% and 99\% for the DC sensor and for the AC sensor respectively), accuracy (99\% for both AC and DC sensors), and reproducibility (99\% for both AC and DC sensors) proved the appropriateness of the sensor for device applications. Additionally, the sensitivity of the laminate anisotropic magnetic field sensor (15 and $1400 \mathrm{mV} / \mathrm{Oe}$ for the DC and AC fields respectively) were the highest stated in the literature for polymer-based ME materials. Such performance, combined with the versatility, flexibility, low cost, light weight, and low temperature production are enormous advantages of developed ME materials for utilization in magnetic sensor device applications [20].

Once key parameters such as accuracy, sensitivity, linearity, resolution and hysteresis have been only unclearly discussed in the literature Reis et al. reported on those performance characteristics on a Metglas/PVDF/Metglas ME laminate [21].

The sensitivity and resolution determined for the AC magnetic field sensor $(992 \mathrm{mV} \cdot \mathrm{Oe}-1$ and 0.3 $\mu \mathrm{Oe})$ and DC magnetic field sensor ( $30 \mathrm{mV} / \mathrm{Oe}$ and $8 \mu \mathrm{Oe})$ were positively comparable with the most sensitive polymer-based ME sensors (see Figure 4). Furthermore, the correlation coefficient, accuracy and linearity obtained values were $0.995,99.4 \%$ and $95.9 \%$ for the DC magnetic field sensor and 0.9998, 99.2\% and 99.4\% and for the ME AC magnetic field sensor. Consequently, the ME materials developed in such work can be used for pioneering AC/DC magnetic field sensors device applications [21]. 
Figure 4. DC magnetic field sensor characterization: (a) linearity, (b) resolution and sensitivity (c) accuracy and (d) hysteresis. Figure taken with permission from [21].
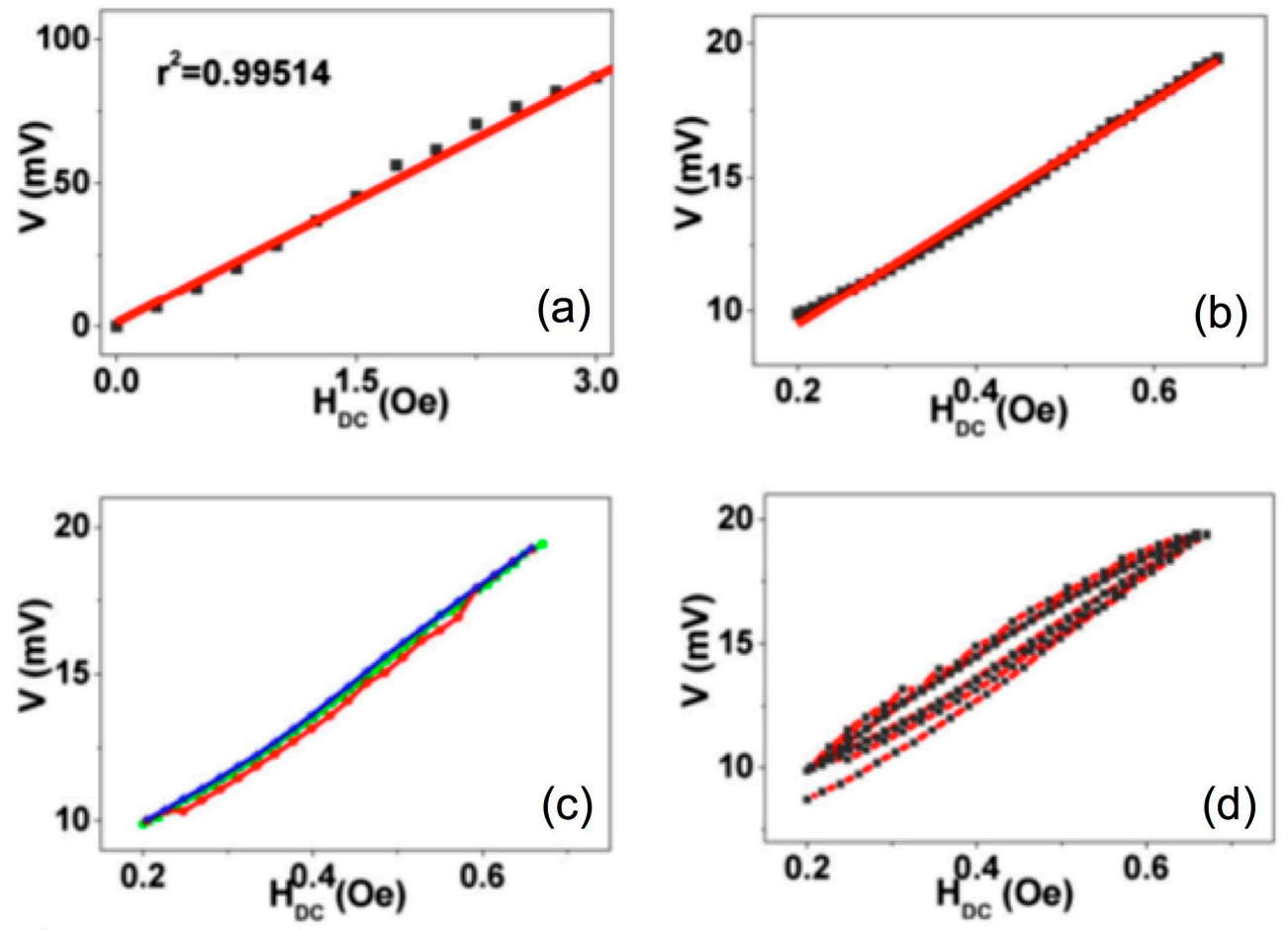

Taking advantage of the same materials and incorporating a charge amplifier, an AC-RMS converter and a microcontroller on chip peripheric analogue to digital converter (ADC) it was developed of a DC magnetic field sensor with readout electronics (see Figure 5, adapted from [22]).

Figure 5. (left) ME signal modulation circuit with two distinct stages: i) charge amplification resulting in an AC voltage output signal; ii) AC-to-DC voltage converter. (right) Analog-to-digital converter circuit to which the previous analogue circuit was connected.
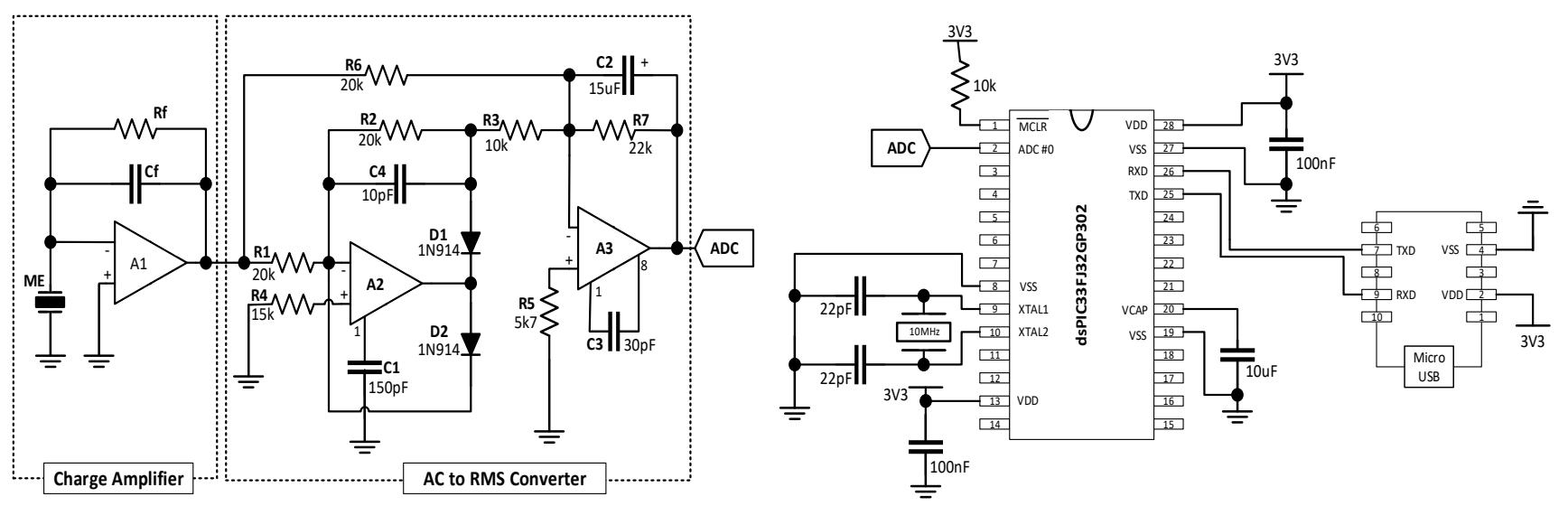

The ME voltage output was not distorted, the linearity was preserved and the ME voltage response was found to increase to $3.3 \mathrm{~V}\left(\alpha_{33}=1000 \mathrm{~V} / \mathrm{cm} . \mathrm{Oe}\right)$ with the introduction of the electronic components. The sensing device, including the readout electronics, revealed a maximum drift of 0.12 Oe with an average total drift of $0.04 \mathrm{Oe}$, a $70 \mathrm{nT}$ resolution and a sensitivity of $1.5 \mathrm{~V} / \mathrm{Oe}$. Such performance was for the first time reported on a polymer-based ME device and was favorably comparable with a reference Hall sensor that showed a maximum drift of 0.07 Oe and an average error 
of $0.16 \mathrm{Oe}, 5 \mathrm{~V} / \mathrm{T}$ sensitivity and $2 \mu \mathrm{T}$ resolution. Such device performance associated to the precise HDC fields measurement mark this polymer-based device as very attractive for device applications such as digital compasses, Earth magnetic field sensing, magnetic field anomaly detectors and navigation, among others.

\subsection{Energy Harvesters}

The use of polymer based ME laminated composites as energy harvesting devices has increased in the last years $[23,24]$. These type of harvesters are usually fabricated with ferromagnetic metallic glasses as the magnetostrictive constituent and the polyvinylidenefluoride (PVDF), not only because of their high ME response, but also due to their excellent mechanical properties and low cost of production [25]. Also, the performance of such energy harvesting device is proportional to the induced ME voltage, and so its best performance will be achieved at the DC magnetic field needed for the maximum ME coupling. The low DC magnetic field required for the polymer based ME laminated composites greatly simplifies the implementation of our ME laminated composites, in comparison with other ME energy harvesting devices, as it will be discussed in the following.

Due to the ME effect measurement process used for our research groups, to rectify the AC signal coming from the ME laminates and convert it into a DC one turns out to be the first important step to be solved. There are in the literature many circuits for this purpose that can be used and applied to energy harvesters. In fact, the output power showed by the laminates will depend on the characteristics of the circuit used, a key factor which makes necessary its optimization. Four are the most common energy harvesting circuits: a full-wave bridge voltage rectifier, two Cockcroft-Walton voltage multipliers with one and two stages and a three stages Dickson voltage multiplier.

The full-wave bridge voltage rectifier circuit is widely used in energy harvesting systems that converts AC voltage to DC voltage [26,27]. The main advantages of this circuit are the low energy loss, low complexity and high efficiency. This circuit consists of four Schottky diodes (see Figure 6 (a)) which convert the AC output voltage of the ME laminate into a DC one through the two half cycles (positive and negative).

The voltage multipliers efficiently convert the AC signal into a DC one and simultaneously increase the output voltage [28]. The Cockcroft-Walton circuit is a half-wave rectifier constituted by $\mathrm{n}$ stages, each stage formed by two diodes and two capacitors (see Figure 6(b) and (c)).

Figure 6. Schematic representation of a Full-wave bridge voltage rectifier (a), one-stage Cockcroft-Walton voltage multiplier (b), two-stages Cockcroft-Walton voltage multiplier (c) and three stages Dickson voltage multiplier (d). $V_{M E}$ represents the induced voltage in the ME laminate, $V_{D}$ represents forward voltage drop across each diode and $V_{\text {Load }}$ measured voltage at the load resistance. Figure adapted from reference [11], with permission of the author. 
(a)

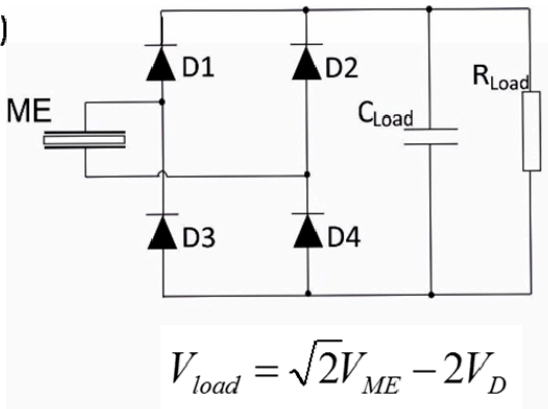

(c)

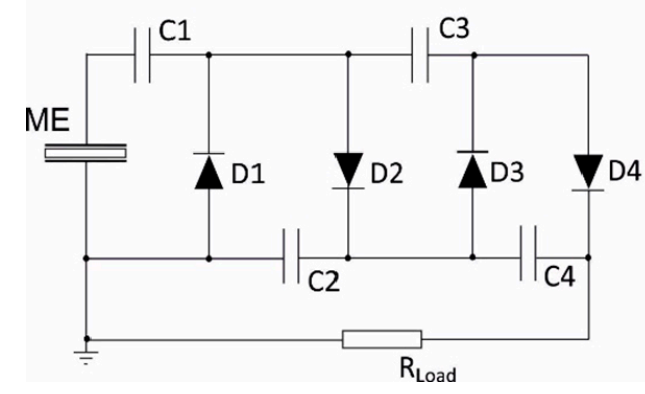

$V_{\text {load }}=\left(4 \sqrt{2} V_{M E}\right)-4 V_{D}$ (b)

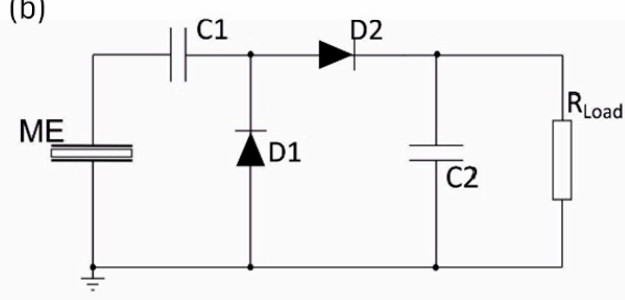

$V_{\text {load }}=\left(2 \sqrt{2} V_{M E}\right)-2 V_{D}$

(d)

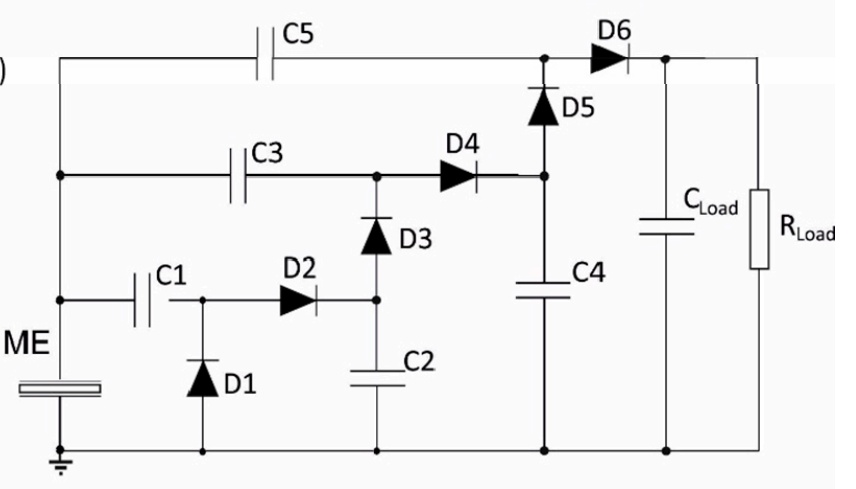

$V_{\text {load }}=\left(6 \sqrt{2} V_{M E}\right)-6 V_{D}$

The one-stage Cockcroft-Walton voltage multiplier (see Figure 6 (b)) consists on a clamper constituted by the capacitor $\mathrm{C} 1$ and the diode D1 and a peak detector constituted by the capacitor $\mathrm{C} 2$ and the diode D2. The clamper signal is measured in the diode D1 and corresponds to the wave input shifted from the negative peak to zero. The peak detector assigns a DC voltage with approximately twice the input peak voltage value. The two-stage Cockcroft-Walton voltage multiplier (see Figure 6 (c)) has a similar behavior than the previous one but the input signal is increased four times by adding another multiplier level.

The Dickson voltage multiplier circuit is also a half-wave rectifier, which can be constructed with $\mathrm{n}$ stages, being each one formed by two diodes and two capacitors. The Dickson multiplier showed in Fig. S3.2.1, (d) is a three-stage circuit based on the original Dickson charge pump, a DC-DC converter where the original DC input is shunted to the ground level and the logic control is replaced by the AC input signal to be harvested [29]. Extensive information about all circuits together with component values can be found in [27].

These four different circuits were tested using a $3 \mathrm{~cm}$ long $\mathrm{Fe}_{64} \mathrm{Co}{ }_{17} \mathrm{Si}_{6.6} \mathrm{~B}_{12.4} / \mathrm{PVDF} /$ $\mathrm{Fe}_{64} \mathrm{Co}_{17} \mathrm{Si}_{6.6} \mathrm{~B}_{12.4}$ three-layered $\mathrm{ME}$ laminate, working at the $\mathrm{L}-\mathrm{T}$ configuration and at its magnetoelastic resonance frequency, measured to be $41.6 \mathrm{kHz}$. The total cross section of the ME laminate is $2.5 \times 0.078 \mathrm{~mm}^{2}$ and the metallic glasses have been previously annealed at $300{ }^{\circ} \mathrm{C}$ for 10 minutes in order to release the internal stresses arisen in their fabrication.

The obtained output ME voltage was continuously monitored by a Hewlett Packard 54603 oscilloscope and subsequently the corresponding output electric power was obtained when varying the load resistance from $1 \mathrm{k} \Omega$ to $1 \mathrm{M} \Omega$.

Figure 7. The output electric power as a function of the load resistance for all the studied energy harvesting circuits. 


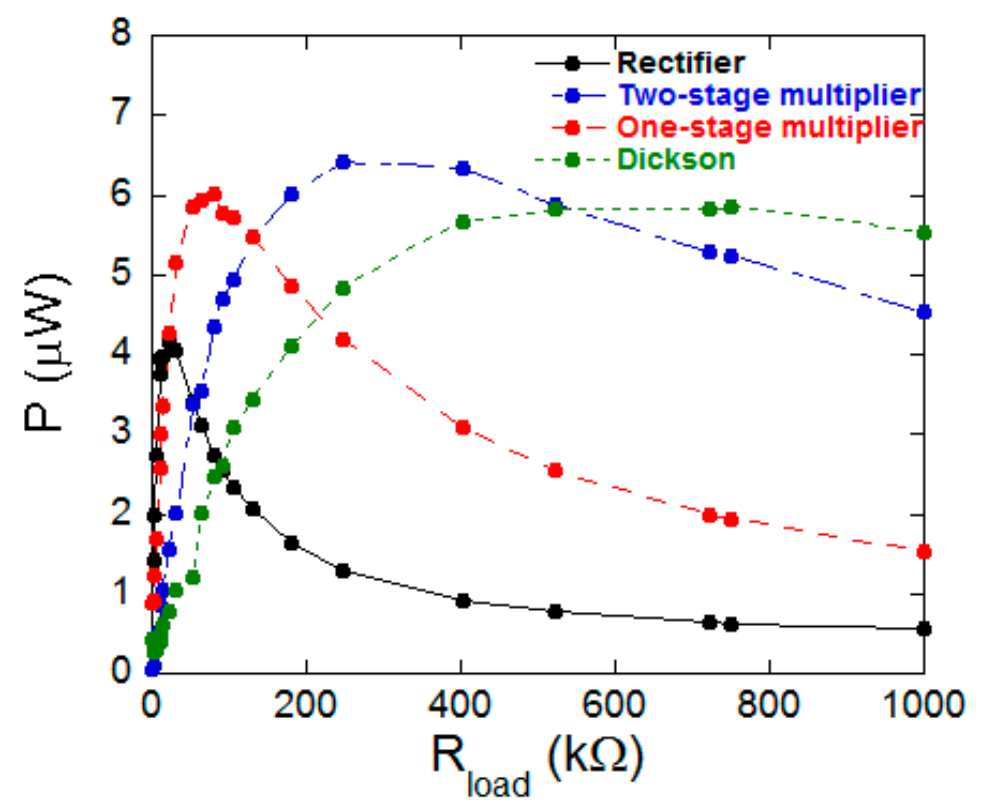

As it can be observed, the maximum output electric power $(6.4 \mu \mathrm{W})$ is achieved for the two-stage multiplier circuit when a load resistance of $250 \mathrm{k} \Omega$ is used. Considering the total volume of the laminate, the corresponding power density value has been estimated to be $1.2 \mathrm{~mW} / \mathrm{cm}^{3}$. It is remarkable that this obtained maximum (magnetoelectric) power generated value is comparable to some previously reported power densities for laminates containing PZT and PVDF (see Table 2) as piezoelectric constituents.

Table 2. Comparison of some power output and power densities values reported so far for some ME laminates. $f_{r}, H_{a c}$ and $H^{*}$ refer to the resonance (or working) frequency, the AC excitacion field and DC field, respectively, for the maximum ME coupling. Table adapted from [23] with permission of the authors.

\begin{tabular}{|c|c|c|c|c|c|}
\hline $\mathbf{f}_{\mathbf{r}}(\mathbf{k H z})$ & $\mathbf{H}_{\mathbf{a c}}(\mathbf{O e})$ & $\mathbf{H *}(\mathbf{O e})$ & $\mathbf{P}(\boldsymbol{\mu W})$ & $\mathbf{P} / \mathbf{V}\left(\mathbf{m W} / \mathbf{c m}^{\mathbf{3}}\right)$ & Materials involved \\
\hline 41.6 & 0.45 & 4.7 & 6.4 & $1.2[23]$ & $\mathrm{Fe}_{64} \mathrm{Co}_{17} \mathrm{Si}_{6.6} \mathrm{~B}_{12.4}$ and PVDF \\
\hline 66.1 & 1 & 66.1 & 0.065 & 0.7 Ref. [30] & $\mathrm{Fe}_{0.7} \mathrm{Ga}_{0.3}$ and PZT \\
\hline 26.9 & 0.3 & 50 & 917.7 & 0.956 Ref. [31] & FeNi and PZT \\
\hline 27.0 & 1 & 800 & 20 & 0.12 Ref. [32] & Terfenol-D and PZT \\
\hline & & & 93.6 & 0.1579 Ref. [33] & PVDF and PZT \\
\hline & & & 75000 & 0.0051 Ref. [34] & APC 855 (piezoelectric) \\
\hline
\end{tabular}

Therefore, the used two-stage multiplier circuit working with a high performance ME device could act as simple and low cost ME effect based energy harvester with good output electric power response. It has to highlight that the obtained output value is within the ultra low-power consumption devices suitable for biomedical wireless communications systems [35], among others.

\section{Size effects on the induced magnetoelectric signal}

When dealing with applications, not only the good bonding between the piezoelectric and magnetostrictive constituents plays an important role, but also other factors as size and relative 
geometry of the components are of great importance [36]. In the following we will focus on these aspects that as it will be shown strongly affects the magnetoelectric laminates performance.

\subsection{Size effects}

Our first study was directed to study the influence of the relative size of the magnetostrictive and piezoelectric elements on the ME response [37]. To do this, rectangular pieces of magnetostrictive Vitrovac $4040\left(\mathrm{Fe}_{39} \mathrm{Ni}_{39} \mathrm{Mo}_{4} \mathrm{Si}_{6} \mathrm{~B}_{12}\right)$ and poled $\beta$-PVDF where cut in several different widths and lengths. In this way, ME laminates with different longitudinal size aspect (LAR) ratio and different transversal size aspect (TAR) ratio between the PVDF and Vitrovac layers were fabricated (see Figure 8).

The obtained results clearly indicated that the ME induced voltage increases with decreasing the LAR ratio value, while laminates with the lowest TAR resulted in better ME performance when compared with higher TAR ratio values. Both those aspect rations, even if being different, further demonstrated a clear correlation with the quantity AreaPVDF / Areavitrovac $=A_{P} / A_{M}:$ the ME response was always optimized for values $A_{P} / A_{M} \approx 1$.

Figure 8. Graphical definition of the longitudinal size aspect (LAR) ratio and transversal size aspect (TAR) ratio between the PVDF and Vitrovac layers.

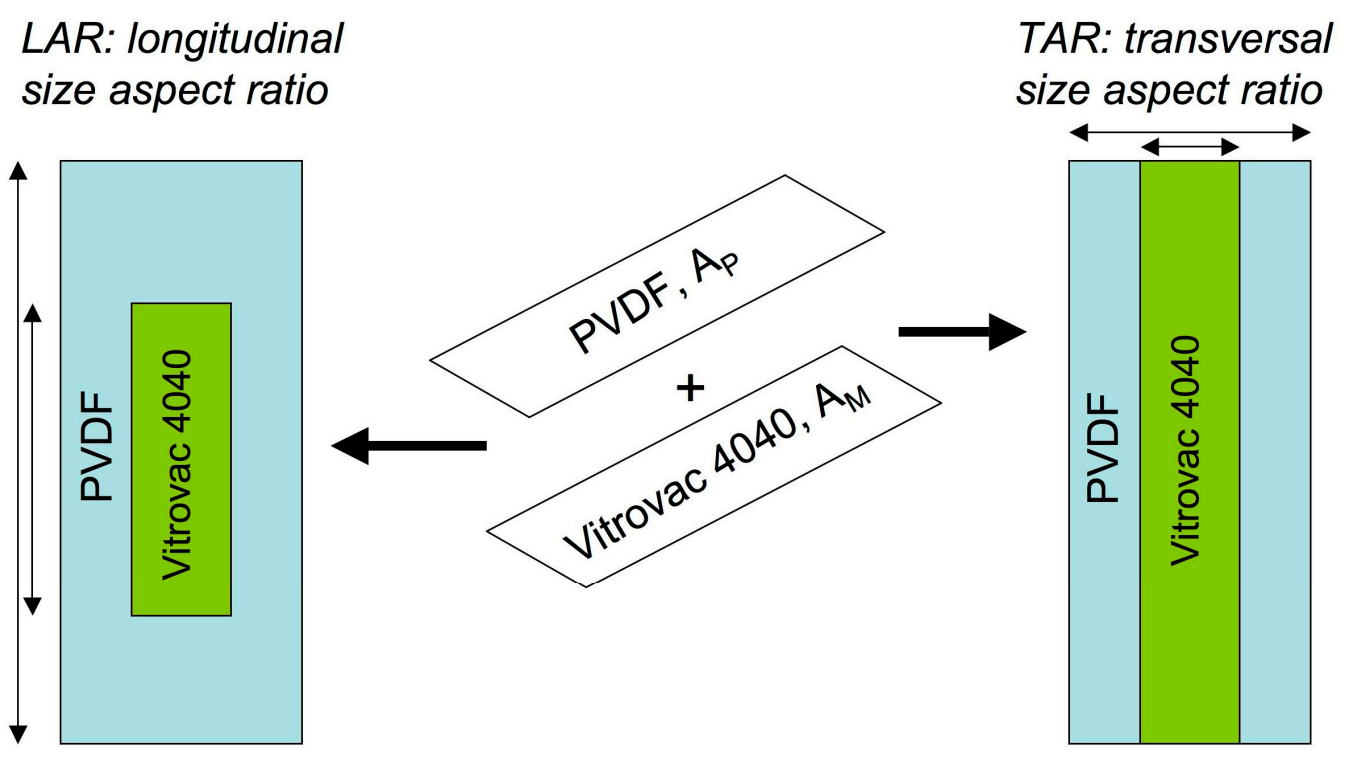

Following this line of work, we proceeded to deeply investigate the case of ME laminates with equal sizes of piezoelectric and magnetostrictive constituents (value $A_{P} / A_{M} \approx 1$ ) and different length values of the laminates. To do this, we fabricated 3, 2, 1 and $0.5 \mathrm{~cm}$ long, three-layered LongitudinalTransverse (L-T) structures of PVDF located between two magnetostrictive ribbons of composition $\mathrm{Fe}_{61.6} \mathrm{Co}_{16.4} \mathrm{Si}_{10.8} \mathrm{~B}_{11.2}$, and the ME response was studied [38].

The first observation, as expected, is that the shorter the laminate, the higher the working frequency of the device, since this frequency matches with the magnetoelastic resonance (MER) frequency of the magnetostrictive constituent $(49.1 \mathrm{kHz}, 70.8 \mathrm{kHz}, 165.5 \mathrm{kHz}$ and $303.7 \mathrm{kHz}$ for the $3,2,1$ and $0.5 \mathrm{~cm}$ long laminates, respectively). Concerning the loss of the ME signal arising from the increase of this working frequency, it is originated by both the magnetostrictive and piezoelectric constituents, but has been quantified to be small enough to be neglected. 
As a direct consequence, the observed loss of the induced ME signal must be inherent to the decrease of the size of the laminate. In fact and for the magnetostrictive constituent, demagnetizing effects can not be neglected, since they become stronger as the length of the magnetostrictive ribbon used decreases. Even if demagnetizing factors for three-layer laminated composites have been already theoretically quantified [39], when the thickness of the piezoelectric layer is very thin, we could calculate the demagnetizing factor of the whole laminated composite by using the following expression:

$$
N_{\text {lam }} \approx \frac{3}{2}<N>
$$

by considering and averaged value $\left\langle N>=1 / 2\left(N_{\text {exp }}+N_{\text {geo }}\right)\right.$ that takes into account values obtained for the experimental [40] and geometrical [41] demagnetizing factors, respectively. The $\langle N\rangle$ value thus obtained has demonstrated to be a good approximation to the real demagnetizing factor for our layered $\mathrm{ME}$ composites. Now and from the estimated demagnetizing factors for the different lengths laminates, we can quantify the loss in the ME signal due to the demagnetizing fields, through the definition of the reduction factor $(R F)$ corresponding to each laminate [40,42]:

$$
R F=\frac{1}{1+N_{\text {lam }} \chi}=\frac{\alpha_{M E}\left(N_{\text {lam }}\right)}{\alpha_{M E}(0)}
$$

where $N_{\text {lam }}$ is the demagnetizing factor of the laminate, $\chi$ is the intrinsic magnetic susceptibility of the ferromagnetic alloy and $\alpha_{M E}(0)$ is the intrinsic ME coefficient (that is, values corresponding to a very long, infinite ribbon and laminate, respectively, not affected by the demagnetizing fields, $N_{\text {lam }}=0$ ) and $\alpha_{M E}\left(N_{\text {lam }}\right)$ is the extrinsic or experimentally measured ME coefficient. In our case, this reduction factor gives the ratio of the ME voltage that is useful respect to the intrinsic ME coefficient, under consideration of demagnetizing fields. Notice that the $\mathrm{ME}$ intrinsic value $\alpha_{M E}(0)$ represents the maximum induced ME voltage that can be extracted from our laminates, independently of their length.

Figure 9. (left) Measured or effective $\mathrm{ME}$ coefficient of the $\mathrm{L}=3 \mathrm{~cm}$ and $1 \mathrm{~cm}$ length laminates and (right) the corresponding intrinsic ones, both represented as a function of the applied magnetic field.

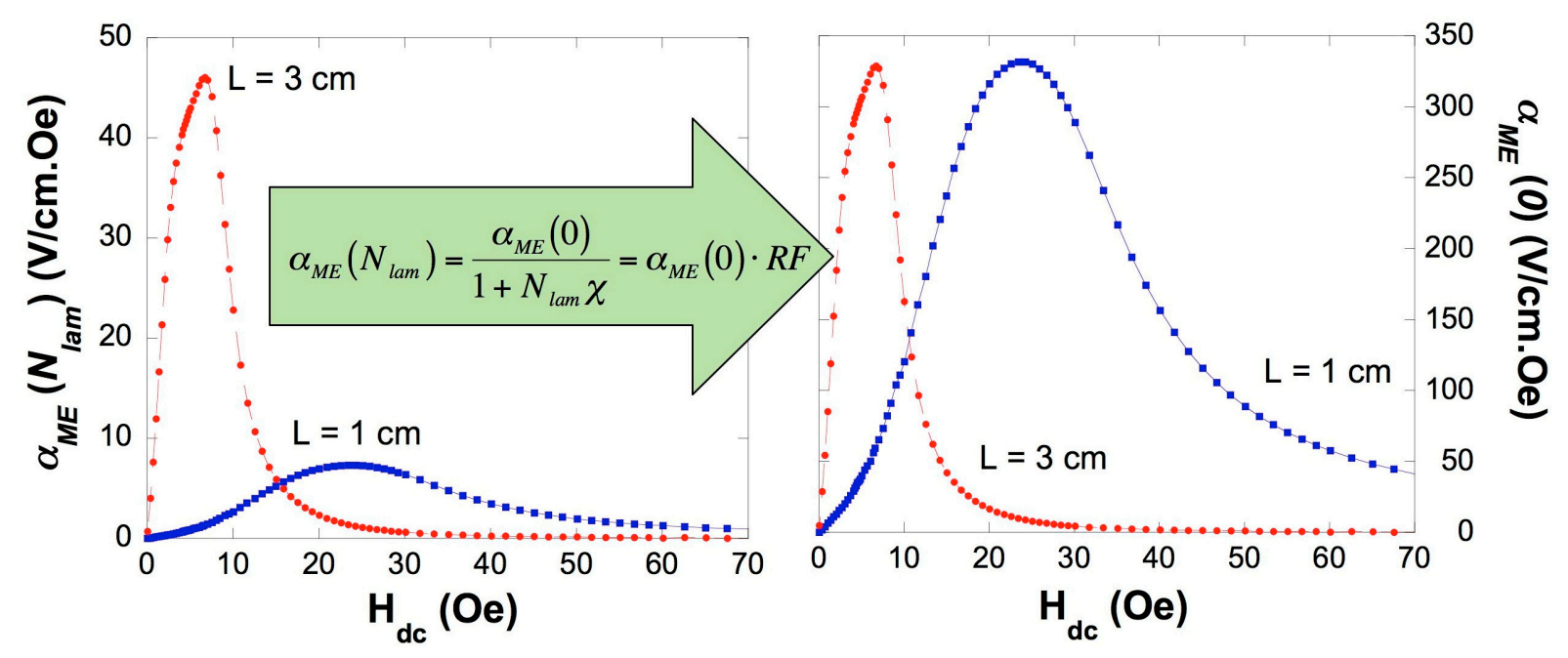


Thus, all estimated intrinsic values should be the same for the different length ME laminates, as can be directly seen in Figure 9. Nevertheless the goodness of the estimation made for the ME intrinsic value giving about $325 \mathrm{~V} / \mathrm{cm}$.Oe, it has also to be pointed that a simple calculation for the reduction factor $R F$ gives for the $3 \mathrm{~cm}$ long laminate losses of about an $87 \%$ of the expected total ME voltage, and this loss value increases as reducing the length of the laminates, reaching the $99 \%$ for the $0.5 \mathrm{~cm}$ long one.

\subsection{Quick and direct comparison of the performance of different ME laminates}

In many cases and in front of ME laminates different with different constituents, one has to afford the task to determine quickly which one is going to offer the best performance at working. When dealing with different devices of the metallic glass/PVDF/metallic glass type (that is with different magnetostrictive constituent but the same PVDF piezoelectric constituent, and also different lengths) in order to have a quick and first idea about how well each ME laminate will work, we can use the so called figure of merit, FM, that characterize the magnetic performance of the magnetostrictive material in such ME devices. This figure of merit is defined as follows [43]:

$$
F M=\lambda_{s} \chi
$$

Thus, taking into account our previous values of the measured susceptibility and magnetostriction, we can estimate the FM value for each used magnetostrictive ribbon, as sumarized in Table 3.

Table 3. Value of the figure of merit of each used magnetostrictive ribbon $(\mathrm{L}=3 \mathrm{~cm})$ composition, in as quenched state.

\begin{tabular}{|c|c|c|c|c|}
\hline & \multicolumn{2}{|c|}{$\left(\mathrm{Fe}_{0.79} \mathrm{Co}_{0.21}\right)_{75+\mathrm{x}} \mathrm{Si}_{15-1.4 \times \mathrm{B}_{10+0.4 \mathrm{x}}}$} & $\mathrm{Fe} 85-\mathrm{x} \mathrm{Co}_{\mathrm{x}} \mathrm{B}_{15}$ \\
\hline $\mathrm{X}$ & 0 & 3 & 6 & 21 \\
\hline$F M$ & 0.99 & 0.72 & 1.15 & 1.75 \\
\hline
\end{tabular}

Therefore and from all these values we can expect to get better results for ME measurements for the laminates corresponding to the $\mathrm{X}=0 / \mathrm{PVDF}$ system (ribbons in as-queched state) than for the $\mathrm{X}=3 / \mathrm{PVDF}$, despite the higher value of the magnetostriction of this last one. Nevertheless, the $\mathrm{X}=21 / \mathrm{PVDF}$ laminate is expected to show the highest ME response, due to its highest magnetostriction value. To confirm this, we can draw the obtained maximum ME voltages for all the laminates fabricated with our magnetostrictive ribbons, versus their corresponding (calculated) FM values.

Figure 10. Maximum ME voltages of laminates fabricated with different magnetostrictive constituents and also different lengths, as a function of the figure of merit of each metallic glass. Figure modified from reference [11], with permission of the author. 


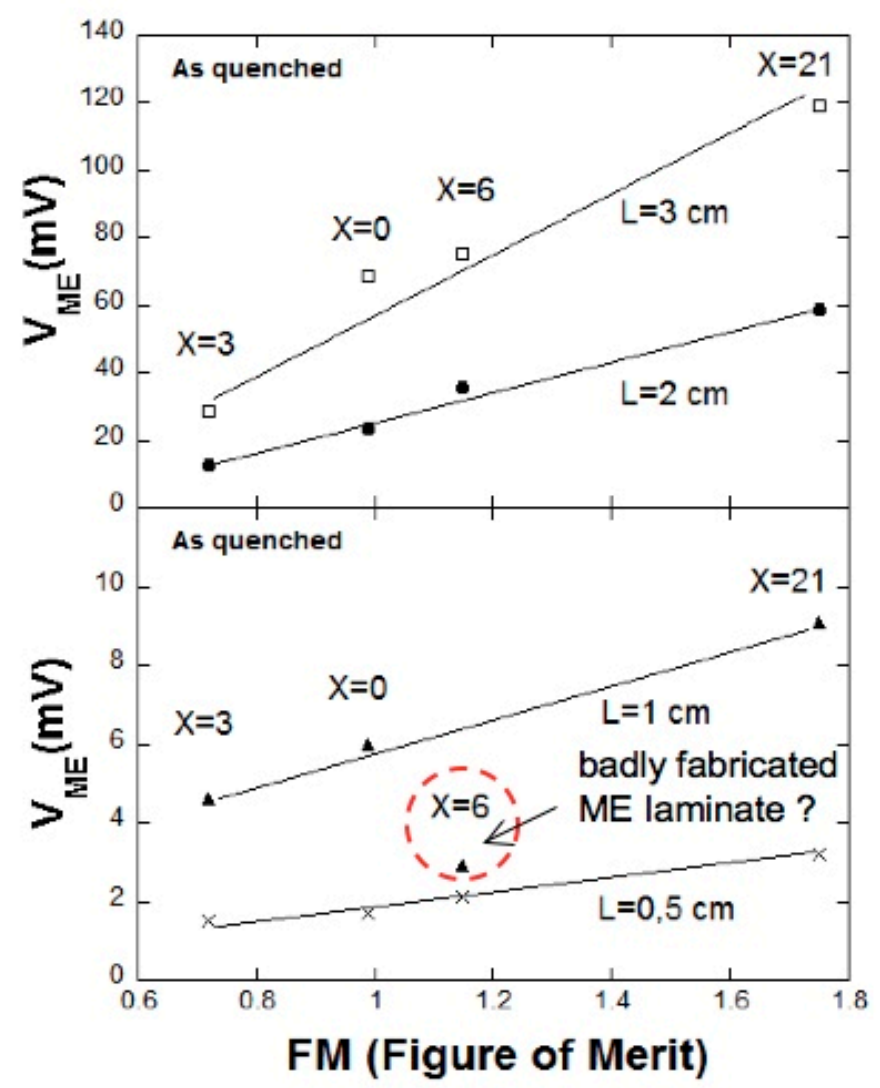

As directly observed, a good linear dependence is obtained which supports also the goodness of the definition given for the $F M$ parameter. Only the $1 \mathrm{~cm}$ long $\mathrm{X}=6 / \mathrm{PVDF}$ laminate fails from this behavior. This disagreement can be attributed to a bad fabrication of the laminate, most probably due to a bad bonding between magnetostrictive and piezoelectric layers.

\section{Futures perspectives: ME laminates for high temperature applications}

The future tasks that ME laminates will have to afford point towards miniaturization and related problems (as previously discussed) and also about working points at high temperatures. Few works have been reported so far concerning the ME effect at high temperatures (see for example [44, 45]) and in most of them piezoelectric ceramic materials as PZT is often used. Collaborating work in ME laminates between Bilbao and Braga laboratories has been mostly carried out by using PVDF (or its copolymer $\mathrm{P}(\mathrm{VDF}-\mathrm{TrFE})$ as piezoelectric constituent. It is important to notice that PVDF shows the $\alpha$ relaxation around $60{ }^{\circ} \mathrm{C}$ and melts $\approx 160{ }^{\circ} \mathrm{C}[46]$, limiting its use in high temperature ME laminates.

Nowadays there is a great interest in synthesizing new piezoelectric polymers for high-temperature applications. Among these new classes of piezopolymers, polyimides have received a lot of interest lately due to their excellent thermal, mechanical and dielectric properties [47-49]. Although investigations in piezoelectric polyimides have only begun recently piezoelectric polyimides have a promising future due to their interesting properties, which are suitable for many applications. Z.Ounaies et al. $[50,51]$ have synthesized a polyimide, $(\beta-\mathrm{CN}) \mathrm{APB} / \mathrm{ODPA}$, containing a single cyano dipolar group $(-\mathrm{CN})$ in the repetitive unit and have shown that polyimides still maintain their piezoelectric properties at higher temperatures than comercial piezopolymers. Detailed information about synthesis, thermal characterization and electric polarization processes can be found in $[49,52]$. We only will remark briefly that its main parameters are a glass transition temperature of $\mathrm{T}_{\mathrm{g}} \approx 200{ }^{\circ} \mathrm{C}$ 
and a degradation temperature of $\mathrm{T}_{\mathrm{d}} \approx 510{ }^{\circ} \mathrm{C}$, temperatures that make these polyimides suitable for high temperature purposes (see Figure $11(l e f t)$ ).

In order to combine the best mechanical and piezoelectric response in the same polymer, copolyimides can be synthesized (see Figure 11 (right)). They are usually obtained by reaction between the dianhydride ODPA and a $50 \%$ mol mixture of two aromatic diamines, namely 1,3-Bis-2cyano-3-(3-aminophenoxy)phenoxybenzene (diamine $2 \mathrm{CN}$, good piezoelectric behaviour) and 1,3Bis(3-aminophenoxy)benzene (diamine $0 \mathrm{CN}$, good mechanical behaviour) in a two-step reaction. Extensive information about synthesis, mechanical and dielectric and properties of these copolyimides can be found in $[54,55]$.

Several test were performed concerning the ME response of such metallic glass / piezopolymer / metallic glass, being metallic glass = Vitrovac 4040 or our home-made amorphous ribbons, and piezopolymer $=\mathrm{PVDF}$ or the $2,6(\beta-\mathrm{CN})$ polyimide or other copolyimides $[56,57]$.

Figure 11. (left) Measured remnant polarization as a function of temperature for commercial PVDF piezoelectric polymer and the 2,6( $\beta-\mathrm{CN}) \mathrm{APB} / \mathrm{ODPA}$ (poli 2,6) polyimide. (right) The remnant polarization of three copolyimides as a function of temperature. Reprinted with permission of the authors from [53]. Copyright 2013 IEEE Xplore.
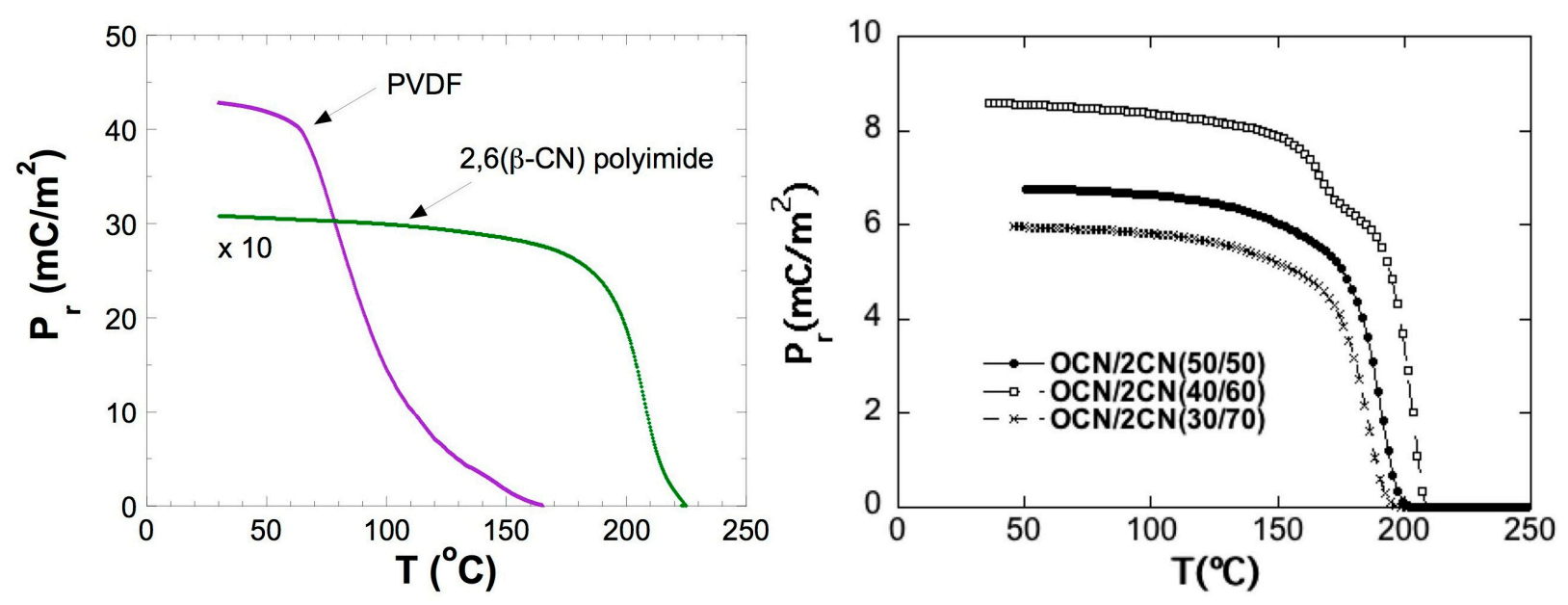

Figure 12. (upper) Dependence of the magnetoelectric coefficient with temperature for (a) $\mathrm{L}=3 \mathrm{~cm} \mathrm{Fe} 61.6 \mathrm{Co} 16.4 \mathrm{Si}_{10.8} \mathrm{~B}_{11.2} / \mathrm{PVDF}$ and $\mathrm{L}=3.7 \mathrm{~cm} \mathrm{Vitrovac}{ }^{\circledR} 4040 /$ poli 2,6 laminated L-T composites. (lower) $\mathrm{L}=1 \mathrm{~cm} \mathrm{Fe} 61.6 \mathrm{Co} 16.4 \mathrm{Si}_{10.8} \mathrm{~B}_{11.2} / \mathrm{PVDF}$ laminated composite. All the measurements were carried out at the magnetoelastic resonance frequency of the laminates. Reprinted with permission of the authors from [57]. Copyright 2013 IEEE Xplore. 


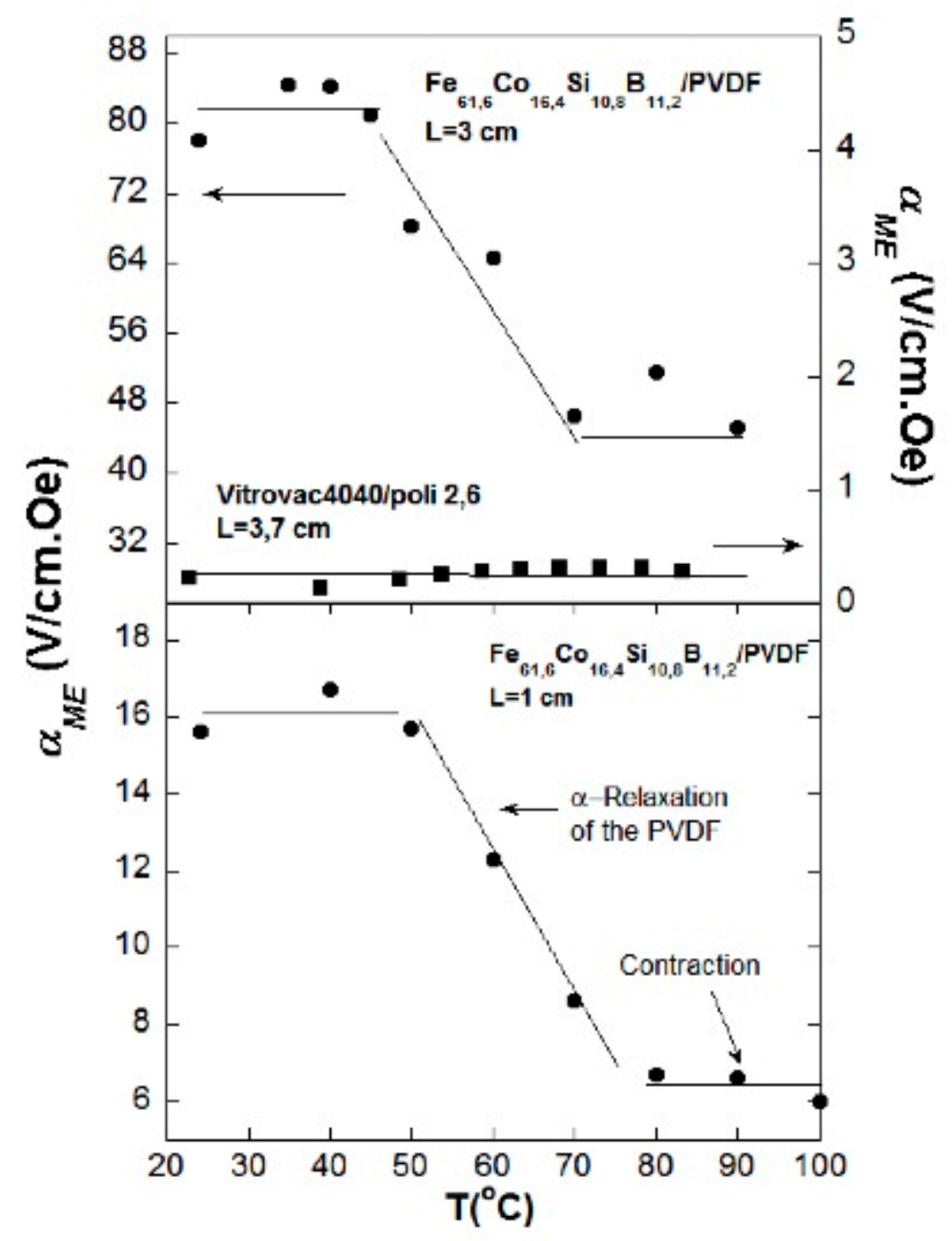

Figure 12 clearly shows that the behavior of the laminates fabricated with PVDF agrees with the temperature dependence of the PVDF polarization value. The change that the measured magnetoelectric coefficient undergoes goes from $82 \mathrm{~V} / \mathrm{cm}$.Oe at room temperature down to 47 $\mathrm{V} / \mathrm{cm}$.Oe at $100{ }^{\circ} \mathrm{C}$ for the $3 \mathrm{~cm}$ long laminate. If we compare this behavior with the laminate fabricated with the high temperature poly 2,6 polyimide, it can be seen that despite the obtained value is much smaller (about $0.5 \mathrm{~V} / \mathrm{cm}$.Oe), the magnetoelectric coefficient keeps constant up to $90{ }^{\circ} \mathrm{C}$, which confirms the great stability that these high temperature poly and copolymides shows at high temperatures. Clearly, a future scope within this line of research hints for the synthesis of high temperature piezoelectric polymers with high value of the remnant polarization (or at least as high as the one of PVDF).

\section{Conclusions/Outlook}

Metallic glass / PVDF magnetoelectric (ME) laminated heterostructures show up to date the highest values achieved for the induced ME voltage (over $300 \mathrm{~V} / \mathrm{cm}$.Oe) despite both constituents, piezopolymeric and magnetic, have not the best piezoelectric and magnetostrictive performance among their respective class of materials. This is due to the fact that the key parameters turn out to be the piezomagnetic value, that in the case of metallic glasses is among the highest ones, and the good 
bonding between magnetostrictive and piezoelectric constituents. Other factors as the relative size of both constituents has been analyzed, giving as conclusion that the best performance is achieved when both components are of equal size. Nevertheless, reduction in the induced ME voltage as shorter the laminated composite is has been quantified, and losses over a $87 \%$ have been determined for laminates of $3 \mathrm{~cm}$ length and below.

When acting as magnetic field sensors, we have shown that our ME laminates work with a sensitivity of $1.5 \mathrm{~V} / \mathrm{Oe}$, a $70 \mathrm{nT}$ resolution and averaged total drift of 0.04 Oe. Such performance can favorably compete with a reference Hall sensor used for comparison. On the other hand, the good capability of our ME devices to work as energy harvesters has been proved. We report a stored power density value for a $3 \mathrm{~cm}$ length ME laminate of about $1.2 \mathrm{~mW} / \mathrm{cm}^{3}$. This (magnetoelectric) power generated value is comparable to previously reported power densities for similar size laminates containing PZT as piezoelectric constituent (with much better piezoelectric performance that PVDF).

Finally, future tendencies point towards the development of new high temperature piezoelectric polymers for applications as directly monitoring of working machinery at the industry or aggressive environments (i.e., the desert, a tunnel or fighting a fire). Polyimides and more specifically, copolymers of the type diamine $2 \mathrm{CN}$ (with good piezoelectric properties) + diamine 0CN (with good mechanical properties) have been tested as a good alternative to PVDF. Despite the modest value of the ME induced voltage measured, this new class of piezoelectric polymers shows an stable behaviour against temperature that makes them promising constituents of ME laminates in future high temperature applications.

\section{Acknowledgments}

J.G., A.L. and J.M.B. would like to thank the financial support from the Basque Government under ACTIMAT and MICRO4FAB projects (Etortek program) and Research Groups IT711-13 project. A. Lasheras wants to thank the Basque Government for financial support under FPI Grant. Technical and human support provided by SGIker (UPV/EHU, MICINN, GV/EJ, ESF) is gratefully acknowledged. P.M., N.P. and S. L.-M. thank the Portuguese Fundação para a Ciência e Tecnologia (FCT) for financial support under Strategic Funding UID/FIS/04650/2013 and project PTDC/EEI-SII/5582/2014, including FEDER funds, UE. P. Martins acknowledges also support from FCT (SFRH/BPD/96227/2013 grant). Financial support from the Spanish Ministry of Economy and Competitiveness (MINECO) through the project MAT2016-76039-C4-3-R (AEI/FEDER, UE) (including the FEDER financial support) is also acknowledged.

\section{Conflicts of Interest}

The authors declare no conflict of interest. 


\section{References}

1- Astrov D.N. Magnetoelectric effect in Chromium Oxide. Soviet Physics Journal of Experimental and Theoretical Physics 1961, 13, 729-733.

2- Rado G.T.; Folen V.J. Observation of the magnetically induced magnetoelectric effect and evidence of antiferromagnetic domains. Physical Review Letters 1961, 7, 310-311.

3- Van Den Boomgard J.; Van Run A.M.J.G.; Van Suchtelen J. Magnetoelectricity in piezoelectricmagnetostrictive composites. Ferroelectrics 1976, 10, 295-298.

4- Lopatin S.; Lopatin I.; Lisnevskaya I. Magnetoelectric PZT/ferrite composite material. Ferroelectrics 1994, 162, 63-68; doi: 10.1080/00150199408245091.

5- Fiebig M. Revival of the magnetoelectric effect. Journal of Physics D: Applied Physics 2005, 38, R123-R152, and references therein; doi:10.1088/0022-3727/38/8/R01

6- Ryu, J.; Vázquez Carazo, A.; Uchino K.; Kim, H.-E.Magnetoelectric properties in piezoelectric and magnetostrictive laminate composites. Japanese Journal of Applied Physics 2001, 40, 49484951 .

7- Zhai J.; Dong S.X.; Xing Z.; Li J.; Viehland D. Giant magnetoelectric effect in metglas/polyvinylidene-fluoride laminates. Applied Physics Letters 2006, 89, 083507(3pp); doi: 10.1063/1.2337996.

8- Van Suchtelen J. Product properties: A new application of composite materials. Philips Research Reports 1972, 27, 28-37.

9- Dong S.X.; Zhai J.; Bai F.; Li J.; Viehland D. Push-pull mode magnetostrictive/piezoelectric laminate composite with an ehanced magnetoelectric voltage coefficient. Applied Physics Letters 2005, 87, 062502 (3pp); doi: 10.1063/1.2007868.

10- Martins P.; Lanceros-Mendez S. Polymer-Based Magnetoelectric Materials. Advanced Functional Materials 2013, 23, 3371-3385; doi: 10.1002/adfm.201202780.

11- Lasheras A. Magnetoelectric metallic glass/polymer laminated composites: from fabrication to applications. Ph D Thesis, University of the Basque Country UPV/EHU, Spain, January 2016.

12- Gutiérrez J.; Barandiarán J.M.; Lasheras A.; Vilas J.L.; San Sebastián M.; León L.M. Resonant response of magnetostrictive/new piezoelectric polymer magnetoelectric laminate. Sensor Letters 2013, 11, 134-137; doi: 10.1166/sl.2013.2769.

13- Kawai H. The Piezoelectricity of Poly(vinylidene Fluoride). Japanese Journal of Applied Physics 1969, 8, 975-976; doi: 10.1143/JJAP.8.975.

14- Silva M.; Reis S.; Lehmann C.S.; Martins P.; Lanceros-Méndez S.; Lasheras A.; Gutiérrez J.; Barandiarán J.M. Optimization of the Magnetoelectric response of poly(vinylidene fluoride) / epoxy / Vitrovac laminates. ACS Applied Materials and Interfaces 2013, 5, 10912-10919; doi: 10.1021/am4031054.

15- Lasheras A.; Gutiérrez J.; Maceiras A.; San Sebastián M.; Barandiarán J.M.; Vilas J.L.; León L.M.; Shiskin D.; Potapov A. Radio Frequency Magnetoelectric Effect Measured at High Temperature. IEEE Transactions on Magnetics 2015, 51, 2500904 (4 pp); doi: 10.1109/TMAG.2015.2390594.

16- Kreitmeier F.; Chashin D. V.; Fetisov Y. K.; Fetisov L. Y.; Schulz I.; Monkman G. J.; Shamonin M. Nonlinear magnetoelectric response of planar ferromagnetic-piezoelectric structures to submillisecond magnetic pulses. Sensors 2012, 12, 14821-14837; doi: 10.3390/s121114821. 
17- Du Tremolet de Laichesserie E. Magnetostriction. Theory and Application of Magnetoelasticity. CRC Press: Boca Ratón, FL, USA, 1993.

18- Gutiérrez, J.; Barandiarán, J.M.; Nielsen O.V. Magnetoelastic properties of some Fe-rich Fe-CoSi-B metallic glasses. Physica Statatus Solidi (a) 1989, 111, 279-283; doi: 10.1002/pssa.2211110129.

19- Gutiérrez J.; Lasheras A.; Barandiarán J.M.; Vilas J.L.; San Sebastián M.; L.M. León. Temperature response of magnetostrictive/piezoelectric polymer magnetoelectric laminates. MRS 2011 Fall Meeting, Boston, USA, November 2011, MRS Online Proceedings Library v. 1398E.

20- Reis S.; Silva M. P.; Castro N.; Correia V.; Gutierrez J.; Lasheras A.; Lanceros-Mendez S.;

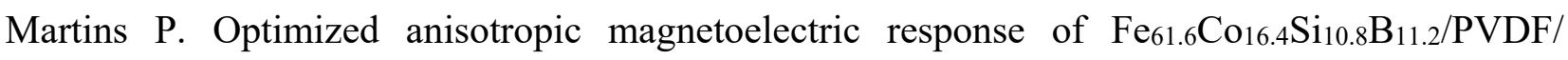

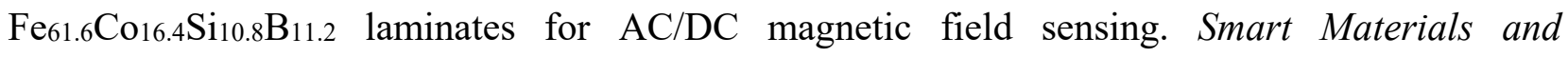
Structures 2016, 25, 055050 (7pp); doi:10.1088/0964-1726/25/5/055050.

21- Reis S.; Silva M. P.; Castro N.; Correia V.; Martins P.; Lasheras A.; Gutiérrez J.; Barandiarán J.M.; Rocha J. G.; Lanceros-Mendez S. Characterization of Metglas/poly(vinylidene fluoride)/Metglas magnetoelectric laminates for AC/DC magnetic sensor applications. Materials and Design 2016, 92, 906-910; doi: 10.1016/j.matdes.2015.12.086.

22- Reis S.; Castro N.; Silva M.P.; Correia V.; Rocha J.G.; Martins P.; Lanceros-Mendez S. Fabrication and characterization of a high-performance polymer-based magnetoelectric DC Magnetic Field sensors, accepted in IEEE Transactions in Industrial Electronics, 2016; doi: 10.1109/TIE.2017.2668989

23- Lasheras, A.; Gutiérrez J.; Reis S.; Sousa D.; Silva M.; Martins P.; Lanceros-Méndez S.; Barandiarán J.M.; Shishkin D.A.; Potapov A.P. Energy harvesting device based on a metallic glass/PVDF magnetoelectric laminated composite. Smart Materials and Structures 2015, 24, 065024 (6 pp); doi: 10.1088/0964-1726/24/6/065024.

24- Reis, S.; Silva M.; Castro N.; Correia V.; Rocha J.G.; Martins P.; Lasheras A.; Gutiérrez J.; Lanceros-Méndez S. Electronic optimization for an energy harvesting system based on magnetoelectric Metglas/poly(vinylidene fluoride)/Metglas composites. Smart Materials and Structures 2016, 25, 085028 (8 pp); doi: 10.1088/0964-1726/25/8/085028.

25- Qi, Y.; Jafferis, N. T.; Lyons, K.; Lee, C. M.; Ahmad, H.; McAlpine, M. C. Piezoelectric ribbons printed onto rubber for flexible energy conversion. Nano Letters 2010, 10, 524-525; doi: $10.1021 / \mathrm{n} 1903377 \mathrm{u}$

26- Li, P.; Wen Y.; Liu P.; Li X.; Jia C. A magnetoelectric energy harvester and management circuit for wireless sensor network. Sensors and Actuators A: Physical 2010, 157, 100-106; doi: 10.1016/j.sna.2009.11.007

27- Tabesh, A.; Fréchette L.G. A Low-Power Stand-Alone Adaptive Circuit for Harvesting Energy From a Piezoelectric Micropower Generator. IEEE Transactions on Industrial Electronics 2010 , 57, 840-849; doi: 10.1109/TIE.2009.2037648.

28- Roscoe, N.M.; Judd M.D. Harvesting Energy From Magnetic Fields to Power Condition Monitoring Sensors. IEEE Sensors Journal 2013, 13, 2263-2270; doi: 10.1109/JSEN.2013.2251625.

29- Barnett, R.E.; Liu J.; Lazar S. A RF to DC Voltage Conversion Model for Multi-Stage Rectifiers in UHF RFID Transponders. IEEE Journal of Solid-State Circuits 2009, 44, 354-370; doi: 10.1109/JSSC.2008.2010991. 
30- Onuta, T.-D; Wang Y.; Long C.J.; Takeuchi I. Energy harvesting properties of all-thin-film multiferroic cantilevers. Applied Physics Letters 2011, 99, 203506 (3 pp); doi: 10.1063/1.3662037.

31- Bian, L.; Wen Y.; Li P.; gao Q.; Zheng M. Magnetoelectric transducer with high quality factor for wireless power receiving. Sensors and Actuators A: Physical 2009, 150, 207-211; doi: 10.1016/j.sna.2009.01.003.

32- Li P.; Wen Y.; Liu P.; Li X.;Jia C. A magnetoelectric harvester and management circuit for wireless sensor networ. Sensors and Actuators A: Physical, 2010, 157, 100-106; doi: 10.1016/j.sna.2009.11.007

33- Vatansever, D.; Hadimani R.L.; Shah T.; Siores E. An investigation of energy harvesting from renewable sources with PVDF and PZT. Smart Materials and Structures 2011, 20, 055019 (6 pp); doi: 10.1088/0964-1726/20/5/055019.

34- Priya, S.; Modeling of electric energy harvesting using piezoelectric windmill. Applied Physics Letters 2005, 87, 184101 (3 pp); doi: 10.1063/1.2119410.

35- Shih Y.-C.; Shen T.; Otis B.P. A $2.3 \mathrm{~mW}$ wireless intraocular pressure/temperature monitor. IEEE Journal of Solid-State Circuits 2011, 46, 2592-2601; doi: 10.1109/JSSC.2011.2164134.

36- Huang Y.; Zhang C.-L. Magnetoelectric effect in a circumferentially polarized composite cylinder. Smart Materials and Structures 2013, 22, 105018 (8 pp); doi: 10.1088/09641726/10/105018.

37- Silva M.P.; Martins P.; Lasheras A.; Gutiérrez J.; Barandiarán J.M.; Lanceros-Mendez S. Size effects on the magnetoelectric response on PVDF / Vitrovac 4040 laminate composites. Journal of Magnetism and Magnetic Materials 2015, 377, 29-33; doi: 10.1016/j.jmmm.2014.10.040.

38- Lasheras A.; Gutiérrez J.; Barandiarán J.M. Quantification of size effects in the magnetoelectric response of metallic glass/PVDF laminates. Applied Physics Letters 2016, 108, 222903 (4 pp); doi: 10.1063/1.4953156.

39- Liverts E.; Grosz A.; Zadov B.; Bichurin M.I.; Pukinskiy Y. J.; Priya S.; Viehland D.; Paperno E. Demagnetizing factors for two parallel ferromagnetic plates and their applicattions to magnetoelectric laminated sensors. Journal of Applied Physics 2011, 109, 07D703; doi: 10.1063/1.3536518.

40- Clark A. E.; Wun-Fogle M. A new method of magnetostrictivity and magnetostriction measurement. IEEE Transactions on Magnetics 1989, 25, 3611-3613; doi: 10.1109/20.42378.

41- Chen D.-X.; Pardo E,; Sanchez A. Demagnetizing factors of rectangular prisms and ellipsoids. IEEE Transactions on Magnetics 2002, 38, 1742-1752; doi: 10.1109/TMAG.2002.1017766.

42- Huong Giang D. T.; Duc P. A.; Ngoc N. T.; Hien N. T.; Duc N. H. Enhancement of the magnetic flux in Metglas/PZT-magnetoelectric integrated 2D geomagnetic device. Journal of Magnetics 2012, 17, 308-315; doi: 10.4283/JMAG.2012.17.4.308.

43- Jia Y.; Zhou W.; Ma K.; Liu Y. Enhanced Magnetoelectric Effect in Permendur/ $\mathrm{Pb}\left(\mathrm{Zr}_{0.52} \mathrm{Ti}_{0.48}\right) \mathrm{O}_{3}$ Laminated Magnetostrictive/Piezoelectric Composite. Applied Sciences 2015, 5, 587-594; doi: 10.3390/app5030587.

44- Burdin D. A.; Chashin D. V.; Ekonomov N. A.; Fetisov Y. K.; Srinivasan G. Multiferroic bending mode resonators and studies on temperature dependence of magnetoelectric interactions. Applied Physics Letters 2012, 100, 242902 (4 pp); doi: 10.1063/1.4729124. 
45- Shen Y.; Gao J.; Wang Y.; Li J.; Viehland D. Thermal stability of magnetoelectric sensors. Applied Physics Letters 2012, 100, 173505 (3 pp); doi: 10.1063/1.4705298.

46- Sencadas V.; Lanceros-Mendez S.; Sabater i Serra R.; Andrio Balado A.; Ribelles J. L. Relaxation dynamics of poly(vinylidene fluoride) studied by dynamical mechanical measurements and dielectric spectroscopy. European Physical Journal E 2012, 35, 41 (11 pp); doi: 10.1140/epje/i2012-12041-x.

47- Fukada E. History and recent progress in piezoelectric polymers. IEEE Transactions on Ultrasonics, Ferroelectrics, and Frequency Control 2000, 47, 1277-1290; doi: 10.1109/58.883516.

48- Fukada E. Recent developments of polar piezoelectric polymers. IEEE Transactions on Dielectrics and Electrical Insulation 2006, 13, 1110-1119; doi: 10.1109/TDEI.2006.247839.

49- Gonzalo, B.; Vilas, J.L.; Breczewski, T.; Pérez-Jubindo, M.A.; De La Fuente, M.R.; Rodriguez, M.; León, L.M. Synthesis, characterization and thermal properties of piezoelectric polyimides. Journal of Polymer Science A: Polymer Chemistry 2009, 47, 722-730; doi: 10.1002/pola.23183; and references therein.

50- Park C.; Ounaies Z.; Su J.; Smith J.G.; Harrison J.S. Polarization stability of amorphous piezoelectric polyimides. MRS Online Proceedings Library Archive v. 600, 1999, 153; doi: 10.1557/PROC-600-153.

51- Park C.; Ounaies Z.; Wise K.E.; Harrison J.S. In situ poling and imidization of amorphous piezoelectric polyimides. Polymer 2004, 45, 5417-5425; doi: 10.1016/j.polymer.2004.05.057.

52- Maceiras A. Polyimides for piezoelectric materials, magnetoelectric nanocomposites and battery separators: synthesis and characterization. $\mathrm{Ph} \mathrm{D}$ Thesis, University of the Basque Country UPV/EHU, Spain, June 2016.

53- Gutiérrez, J.; Lasheras, A.; Barandiarán, J.M.; Vilas, J.L.; San Sebastián, M.; León, L.M. Improving the magnetoelectric response of laminates containing high temperature piezopolymers. IEEE Transactions on Magnetism 2013, 49, 42-45; doi: 10.1109/TMAG.2012.2220125.

54- Gutiérrez, J.; Lasheras, A.; Barandiarán, J.M.; Vilas, J.L.; Maceiras, A.; León, L.M. Improving the performance of high temperature piezopolymers for magnetoelectric applications. Key Engineering Materials 2013, 543, 439-442; doi: 10.4028/www.scientific.net/KEM.543.439.

55- Maceiras, A; Martins, P; San Sebastián, M; Lasheras, A; Silva, M; Laza, J.M; Vilas, J.L; Gutierrez, J; Lanceros-Mendez, S; Barandiaran, J.M; Leon, L.M. Synthesis and characterization of novel piezoelectric nitrile copolyimide films for high temperature sensor applications. Smart Materials and Structures 2014, 23, 105015 (7 pp); doi: 10.1088/0964-1726/23/10/105015.

56- Gutiérrez, J.; Lasheras, A.; Barandiarán, J.M.; Vilas, J.L.; San Sebastián, M.; León, L.M. Temperature response of Magnetostrictive/Piezoelectric Polymer magnetoelectric Laminates. Key Engineering Materials 2012, 495, 351- 354; doi: 10.4028/www.scientific.net/KEM.495.351.

57- Gutiérrez, J.; Lasheras, A.; Maceiras, A.; San Sebastián, M.; Barandiarán, J.M.; Vilas, J.L.; León, L.M; Shishkin, D; Potapov, A. Radiofrequency magnetoelectric effect measured at high temperature. IEEE Transactions on Magnetism 2015, 51, 2500904 (4 pp); doi: 10.1109/TMAG.2015.2390594. 